\title{
Dark Matter Searches from the Sun with the KM3NeT-ORCA detector
}

\author{
The KM3NeT Collaboration \\ $\$$ https://www.km3net.org/km3net-author-list-for-icrc-2019 \\ Email: daniellceugr.es
}

The ORCA (Oscillations Research with Cosmics in the Abyss) detector is currently being deployed in the Mediterranean sea, near Toulon (France). With an instrumented volume of 8 Mton, this water Cherenkov detector is the KM3NeT detector with the highest granularity optimised to observe atmospheric neutrinos in the GeV range. Even though the primary goal of ORCA is to determine the neutrino mass ordering, the detector is also well suited to perform indirect searches for dark matter signals coming from very massive objects like the Sun. A popular set of dark matter candidates are those based on WIMPs (Weakly Interactive Massive Particles). WIMPs decay or annihilate into standard model particles producing also neutrinos which propagate to the detector in the Earth. The ORCA analysis for WIMP searches from the Sun, considering several physics channels, will be presented and the results in terms of sensitivity to neutrino flux, spin-dependent, and spin-independent scattering cross-section reported.

Corresponding authors: D. Lopez-Coto ${ }^{\dagger 1}$, S. Navas ${ }^{\ddagger 1}$, J.D. Zornoza ${ }^{2}$.

${ }^{1}$ Dpto. de Física Teórica y del Cosmos \& C.A.F.P.E., University of Granada, Granada, Spain.

${ }^{2}$ IFIC - Instituto de Física Corpuscular (Univ. de Valéncia - CSIC), E-46980 Paterna, Spain.

36th International Cosmic Ray Conference -ICRC2019-

July 24th - August 1st, 2019

Madison, WI, U.S.A.

\footnotetext{
* for collaboration list see PoS(ICRC2019)1177

${ }^{\dagger}$ Main author.

${ }_{\ddagger}$ Presenter.
} 


\section{Introduction}

Although there are several observations which supply evidence for the existence of dark matter (DM), its detection is, nowadays, one of the most challenging open problems in cosmology, astrophysics and particle physics [1,2] due to its unknown and elusive nature.

Dark matter particles are searched for using different approaches: direct detection of the recoil from collisions with nuclei, production at particle accelerators and indirect detection through the production of secondary particles when they annihilate or decay $[3,4]$. One of the most accepted hypotheses to describe its nature assumes that DM is made of WIMPs (Weakly Interactive Massive Particles) due to the assumption that these particles are a thermal relic of the Big Bang [5]. As massive particles, WIMPs tend to accumulate in massive astrophysical bodies such as the Earth, the Galactic Center, galaxy clusters or the Sun [6].

Most of the WIMP candidates annihilate to pairs and produce Standard Model particles which interact or decay producing neutrinos in the final state. Here it is where neutrino telescopes, like ORCA, come into play. One of the most favoured WIMP candidate is the neutralino $(\chi)$ [7]. Neutralinos can have two types of interactions with ordinary matter: spin-dependent (SD) and spin-independent (SI) [8]. On the one hand, neutralinos couple to the spin of the target nucleus, which mainly happens in interactions with nuclei with an odd number of nucleons. On the other hand, neutralinos couple to the mass of the target nucleus so the sensitivity to this interaction increases for heavier nuclei, which favours direct detection experiments. In the Sun, these two types of interactions are possible since the star contains both light elements with an odd number of nucleons, like hydrogen, and relatively heavy elements, like helium and oxygen. Although the fraction of each of these interactions in the Sun is unknown, it is expected that SD interactions with hydrogen atoms dominate due to the large abundance of this element in the star.

In the analysis presented in this document, the focus has been set in the Sun as a neutrino source [9] from WIMP annihilations [10]. WIMP masses in the range from 15 to $100 \mathrm{GeV}$ and three WIMP annihilations channels $\left(\tau^{+} \tau^{-}, b \bar{b}, W^{+} W^{-}\right)$have been explored (assuming a branching ratio of $100 \%$ to each channel). The analysis corresponds to one year of simulated events with one KM3NeT-ORCA building block (115 detection units). Different quality cuts have been used in order to reduce the atmospheric background and optimise the neutrino flux limits $\Phi_{v_{\mu}+\bar{v}_{\mu}}$ and the spin-dependent $\sigma_{S D}$ and spin-independent $\sigma_{S I}$ WIMP-proton scattering cross-sections.

\section{The ORCA detector}

The KM3NeT-ORCA (ORCA from now on) detector [12] is an undersea neutrino telescope under construction located at $42^{\circ} 48^{\prime} \mathrm{N} 06^{\circ} 02^{\prime} \mathrm{E}$, anchored at a depth of $2450 \mathrm{~m}$ about $40 \mathrm{~km}$ offshore from Toulon (France) and $10 \mathrm{~km}$ west of the site of the existing ANTARES telescope [13, 14]. The full ORCA configuration will consist on 115 detection units with an average horizontal spacing of $20 \mathrm{~m}$. Each detection unit is about $200 \mathrm{~m}$ in height, hosting 18 DOMs (Digital Optical Modules) with a vertical spacing of $9 \mathrm{~m}$ and starting about $40 \mathrm{~m}$ from the sea floor. Each DOM contains 31 PMTs (photo-multipliers tubes), which detect the Cherenkov light yield by secondary charged particles resulting from the high energy neutrino interaction with sea water. The PMTs measuring a signal are determined, each signal ('hit') being characterised by the photon arrival 


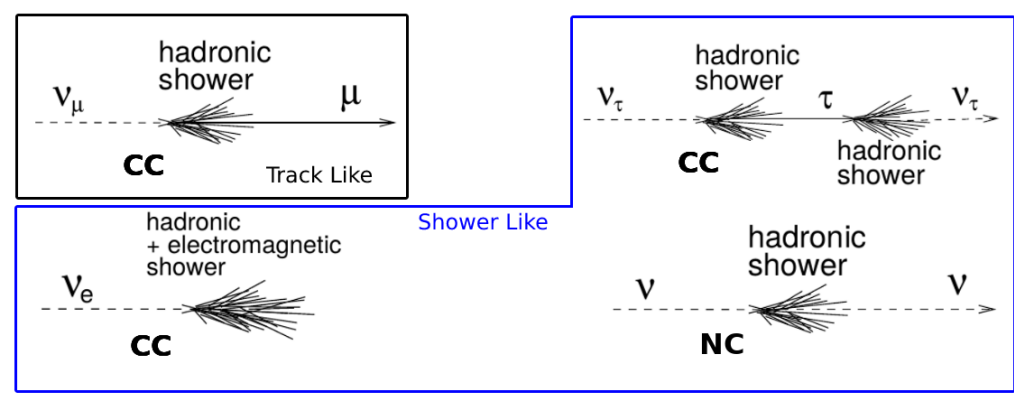

Figure 1: Different neutrino event topologies in neutrino telescopes. A CC interaction of a $v_{\mu}$ (top left) producing an hadronic shower and a $\mu$ track. A CC of a $v_{\tau}$ producing an hadronic shower and a $\tau$ particle which decays producing another hadronic shower and a $v_{\tau}$ (top right). A CC interaction of a $v_{e}$ producing an hadronic and an electromagnetic shower (bottom left). A NC interaction of any neutrino flavour producing hadronic showers (botton right). Figure from [11].

time and the signal amplitude (deposited charge). The hit data are converted to digitised arrival time and time-over-threshold (ToT), i.e. the time the analog signal exceeds a predefined threshold.

Neutrino induced events can be classified into two main groups: track-like and shower-like events. Track-like events are due to charged-current $v_{\mu}$ interactions which produce in the final state muons with track lengths at hundred of meters and trajectories almost colinear with the original neutrino direction. Also $v_{\tau}$ charged-current interactions can produce a high-energy muon in the final state through a muonic decay of the final-state $\tau$ with a branching ratio of about $17 \%$. All neutral-current reactions, as well as charged-current reactions of $v_{e}$ and most $v_{\tau}$, produce showerlike events. These showers are typically several metres long and therefore small compared to inter-DOM distances. The analysis presented in this document is focused on track-like events only. The reconstruction algorithms maximize a likelihood function that the observed space-time PMT hit pattern is consistent with the Cherenkov emission from the fitted muon trajectory. From this reconstruction, several quantities are retrieved such as the interaction vertex, the direction and an estimator of the fit quality. The neutrino angular resolution is better than $10^{\circ}$ for energies relevant for the NMH determination [12]. The classification algorithm is based on a Boosted Decision Tree (BDT) which receives as inputs the characteristics of the signal, time interval between hits, number of hits, number of DOMs/PMTs and charge. Each tree votes if the event is more noise-, track- or atmospheric muon-like and retrieves different labels with values ranging from 0 to 1 meaning that the probability for each event is of one kind. The main cut parameter used to optimise sensitivities is the track score which characterises the event as track-like (values close to 1) or shower-like (values close to 0 ).

\section{Search Method}

The strategy followed in the search of WIMP annihilation in the Sun is based on the maximisation of the following likelihood function $[15,16]$ for track-like events:

$$
\mathcal{L}\left(n_{s}\right)=e^{-\left(n_{s}+N_{\text {out }}\right)} \prod_{i}^{N_{\text {in }}}\left(n_{s} \cdot S\left(\psi_{i}, N_{h i t, i}, \beta_{i}\right)+N_{\text {tot }} \cdot B\left(\psi_{i}, N_{\text {hit }, i}, \beta_{i}\right)\right),
$$




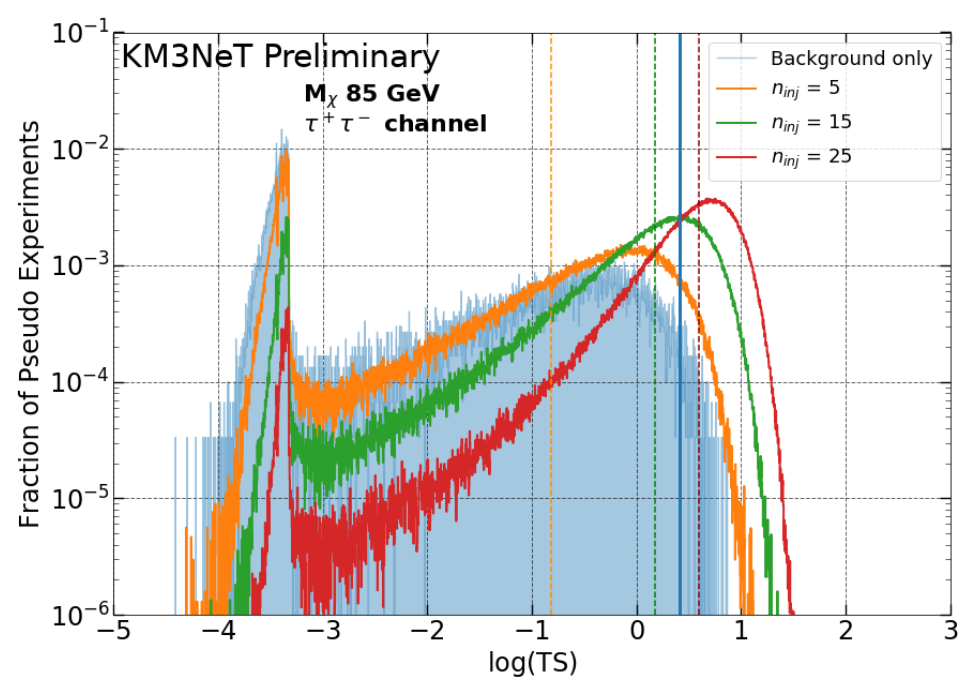

Figure 2: Distribution of the test statistic for a WIMP mass of $85 \mathrm{GeV}$, WIMP-WIMP $\rightarrow \tau^{+} \tau^{-}$ annihilation channel, track score $>0.7$ and different number of injected signal events $n_{i n j}$. The continuos blue vertical line shows where the $90 \%$ of the background-only distribution is, whereas the dashed coloured vertical lines represents the distribution median value for each scenario with different number of injected signal.

where all the information which characterises the signal and background events are contained in the Probability Density Functions for an event to be signal $(S)$ or background $(B) . \psi_{i}$ is the angular distance of the $i$-th event to the Sun, $N_{h i t, i}$ is the number of hits used in the reconstruction of the $i$-th event (used as an energy estimator) and $\beta_{i}$ is the angular error of the $i$-th event. The total number of reconstructed events is denoted by $N_{t o t} . n_{s}$ is the number of expected signal events, it is also the parameter to fit in the likelihood which is only allowed to take values greater than $0 . N_{\text {in }}$ is the number of events within a certain angular distance to the Sun which are considered as possible signal events, $N_{\text {out }}$ is the number of events with an angular distance to the Sun great enough to be discarded as signal and is defined as $N_{\text {out }}=N_{\text {tot }}-N_{\text {in }}$.

The signal and background PDFs can be factorised as follows:

$$
\begin{aligned}
& S\left(\psi_{i}, N_{h i t, i}, \beta_{i}\right)=S_{1}\left(\psi_{i}\right) \cdot S_{2}\left(N_{h i t, i}, \beta_{i}\right), \\
& B\left(\psi_{i}, N_{h i t, i}, \beta_{i}\right)=B_{1}\left(\psi_{i}\right) \cdot B_{2}\left(N_{h i t, i}, \beta_{i}\right),
\end{aligned}
$$

where the independence between the angular distance to the source PDF $\left(S_{1}\left(\psi_{i}\right)\right.$ and $\left.B_{1}\left(\psi_{i}\right)\right)$ and the probability density function of the number of hits and angular error $\left(S_{2}\left(N_{h i t, i}, \beta_{i}\right)\right.$ and $\left.B_{2}\left(N_{h i t, i}, \beta_{i}\right)\right)$ is being assumed.

To compute the signal PDF ingredients we need the neutrino spectra yield by WIMP-WIMP annihilation in the Sun, which was obtained using the package WimpSim 4.1 [10, 17]. The background ingredients were computed using HKKM 2014 flux tables for atmospheric neutrinos [18].

The test statistic TS used here is then defined as the logarithm of the ratio between the likelihood signal plus background and background only scenarios: 


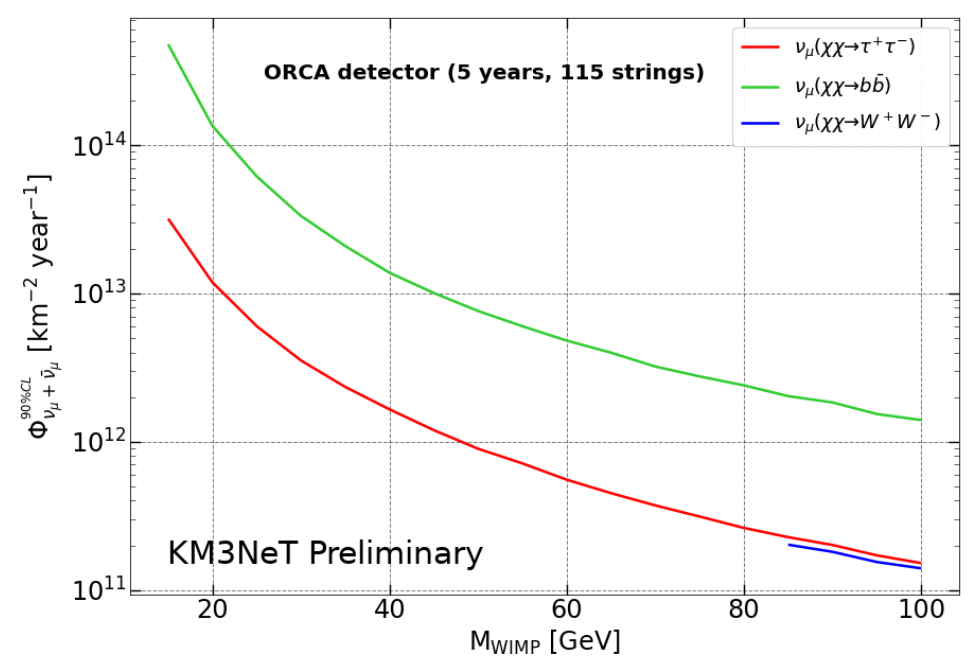

Figure 3: $90 \%$ CL upper limit in the neutrino flux from WIMP annihilations in the Sun as a function of the WIMP masses for three annihilation channels considered.

$$
T S=\log _{10}\left(\frac{\mathcal{L}_{S+B}}{\mathcal{L}_{B}}\right)
$$

where $\mathcal{L}_{S+B}=\mathcal{L}\left(n_{s}=\hat{n}_{S}\right)$ being $\hat{n}_{s}$ the number of expected signal events which optimise the likelihood function, and $\mathcal{L}_{B}=\mathcal{L}\left(n_{s}=0\right)$, i.e. assuming no signal events, for 'background-only' case.

In order to compute the detector sensitivities, the approach given by Neyman [19] is followed. The $90 \%$ confidence level (CL) sensitivity in terms of number of detected events is calculated after building the TS distributions from the pseudo-experiments for different selection cuts, WIMP masses, annihilation channels considered and different number of injected signal events $\left(n_{i n j}=\right.$ $0,1,2,3, \ldots)$, being $n_{i n j}=0$ the background-only scenario where no signal events are present in the simulation.

To mimic the statistical fluctuations, the TS distributions were convolved with a Poissonian distribution, and with a Gaussian distribution for taking into account an assumed 15\% of uncertainty in the number of detected events (Eq. 3.5).

$$
P(T S \mid \mu)=\sum_{i=0}^{n_{\text {inj }}^{\max }} P(T S \mid i) \cdot P(i \mid \mu),
$$

where $P(T S \mid \mu)$ is the new TS distribution after the convolution (fig.2), $P(T S \mid i)$ is the original TS distribution and $P(i \mid \mu)$ is the probability distribution to convolve with, and it is defined as:

$$
P(i \mid \mu)=\int_{\mu-4 \sigma_{\mu}}^{\mu+4 \sigma_{\mu}} P(i \mid \bar{\mu}) \cdot G\left(\bar{\mu} \mid \mu, \sigma_{\mu}\right) d \bar{\mu},
$$

being $n_{i n j}^{\max }$ the maximum number of injected signal events in the pseudo-experiments, $P(i \mid \bar{\mu})$ the poissonian probability to detect $i$ events given a mean of $\bar{\mu}$ detected events, $G\left(\bar{\mu} \mid \mu, \sigma_{\mu}\right)$ the probability to detect $\bar{\mu}$ events given a $\mu$ mean of detected events with an uncertainty $\sigma_{\mu}$.

The sensitivity, or $90 \%$ CL upper limit on neutrino flux (fig.3), is computed as:

$$
\Phi_{v_{\mu}+\bar{v}_{\mu}}^{90 \% C L}\left(M_{\mathrm{WIMP}}\right)=\frac{\mu_{90 \% C L}\left(M_{\mathrm{WIMP}}\right)}{\operatorname{Acc}\left(M_{\mathrm{WIMP}}\right) \cdot T_{\mathrm{eff}}},
$$


where $\mu_{90 \% C L}$ is the median upper limit defined as the minimum number of detected neutrino events needed to be able to distinguish signal from background with a 90\% CL in 50\% of the experiments, $T_{\text {eff }}$ is the effective detector data taking period and $\operatorname{Acc}\left(M_{\mathrm{WIMP}}\right)$ is the detector acceptance, which is obtained averaging the detector effective area $\left(A_{\text {eff }}\right)$ with the neutrino spectra yield by WIMPWIMP annihilation:

$$
\operatorname{Acc}\left(M_{\mathrm{WIMP}}\right)=\overline{A_{\mathrm{eff}}}\left(M_{\mathrm{WIMP}}\right)=\frac{\sum_{j=\bar{v}_{\mu}, \bar{v}_{\mu}}\left(\int_{0}^{M_{\mathrm{WIMP}}} A_{\mathrm{eff}}^{j}\left(E_{j}\right) \frac{d N_{j}}{d E_{j}} d E_{j}\right)}{\int_{0}^{M_{\mathrm{WIMP}}} \frac{d N_{v_{\mu}}}{d E_{v_{\mu}}} d E_{v_{\mu}}+\frac{d N_{\bar{v}_{\mu}}}{d E_{\bar{v}_{\mu}}} d E_{\bar{v}_{\mu}}},
$$

where $j$ stands for neutrino or anti-neutrino in the summation and $\frac{d N_{v_{\mu}\left(\bar{v}_{\mu}\right)}}{d E_{v_{\mu}}\left(\bar{v}_{\mu}\right)}$ is the neutrino (antineutrino) spectra yield by WIMP-WIMP annihilation for a given channel.

\section{Results}

The best sensitivity (fig.3) is achieved for the following cuts: events reconstructed as up-going neutrinos $\left(\theta_{\text {zenith }}>90^{\circ}\right)$, track-score $>0.7$, noise-like events $<0.05$ and atmospheric muon-like events $<0.1$. The flux limits can be translated into cross-section limits through a conversion factor $k_{S D(S I)}^{f}{ }^{1}[20]:$

$$
\sigma_{S D(S I)}\left(M_{\mathrm{WIMP}}\right)=k_{S D(S I)}^{f}\left(M_{\mathrm{WIMP}}\right) \cdot \Phi_{v_{\mu}+\bar{v}_{\mu}}^{f}\left(M_{\mathrm{WIMP}}\right),
$$

where $\sigma_{S D(S I)}$ is the spin-dependent (spin-independent) scattering cross-section. $\Phi_{v_{\mu}+\bar{v}_{\mu}}^{f}\left(M_{\mathrm{WIMP}}\right)$ is the sensitivity to neutrino flux computed using Eq. 3.7. $k_{S D(S I)}^{f}\left(M_{\mathrm{WIMP}}\right)$ is the conversion factor between flux and cross-section for a given annihilation channel $f$. This conversion factor was calculated using DARK SUSY 6.1 [21].

\section{Summary and next steps}

The potential of the ORCA neutrino telescope to search for WIMP annihilations in the Sun has been studied and results in terms of limits on the neutrino flux and WIMP-proton scattering cross-section for three annihilation channels (WIMP WIMP $\rightarrow \tau^{+} \tau^{-}, W^{+} W^{-}, b \bar{b}$ ) and WIMP mass ranging from 15 to $100 \mathrm{GeV}$ are computed.

In order to properly compare with other running experiments, an extension to higher WIMP masses considering also the shower-like channel is being carried out.

\section{Acknowledgements}

The authors acknowledge the financial support from the Spanish Ministerio de Ciencia, Innovación y Universidades: Programa Estatal de Generación de Conocmiento, ref. PGC2018-096663B-C44, FPA2015-65150-C3-3-P (MCIU/FEDER).

\footnotetext{
${ }^{1}$ For more details about this conversion factor see [20].
} 

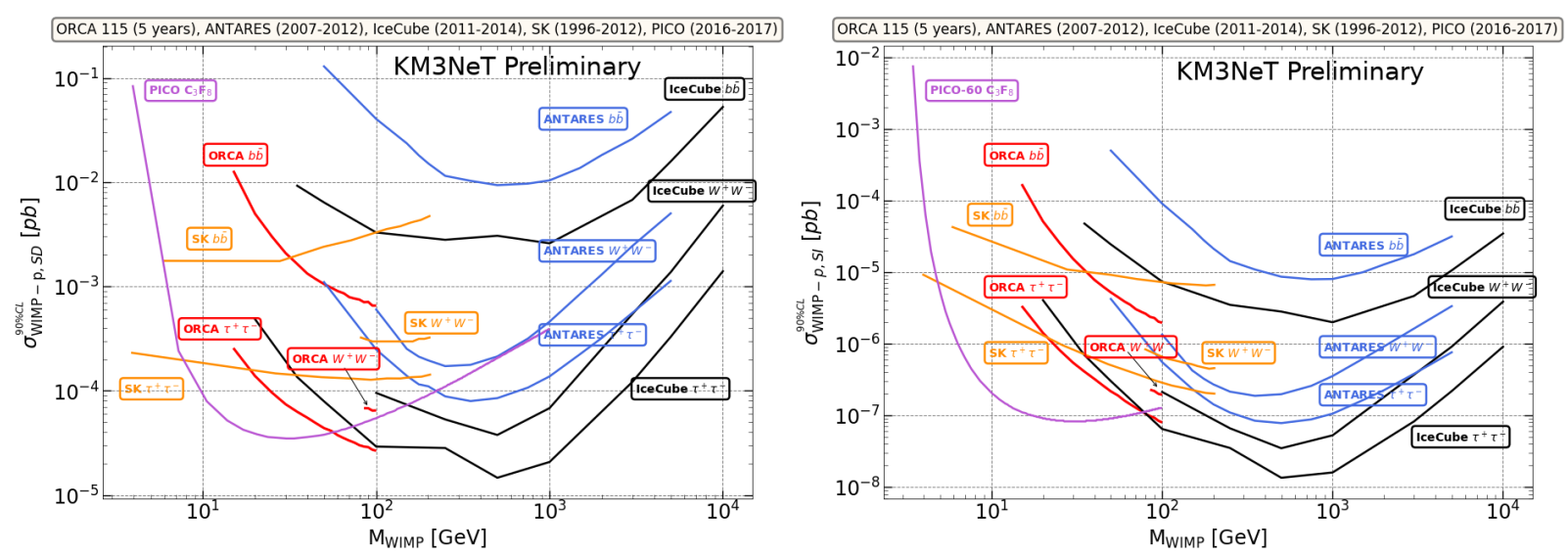

Figure 4: WIMP-proton SD (left) and SI (right) scattering cross-sections limits as a function of WIMP mass for the three annihilation channels considered. Comparative between 5 years of ORCA simulated data, 5 years of ANTARES data [16], Ice Cube 3 years of data [22], Super Kamiokande 16 years [23] and $\mathrm{PICO}-60 \mathrm{C}_{3} \mathrm{~F}_{8}$ [24] 1 year.

\section{References}

[1] G. Bertone et al., Particle Dark Matter: Observations, Models and Searches, Cambridge University Press, Cambridge 2010.

[2] D. Majumdar, Dark Matter: An Introduction, CRC Press, Boca Raton 2014.

[3] M. Tanabashi et al., Particle Data Group, Phys. Rev. D 98 (2018) 030001.

[4] J. M. Gaskins, A review of indirect searches for particle dark matter, Contemp. Phys. 57 (2016) no. 4, 496 [arXiv:1604.00014 [astro-ph.HE]].

[5] K. Griest and P. Murdin, WIMP and MACHOs, (2006) Encyclopedia of Astronomy \& Astrophysics, IOP Publishing Ltd.

[6] W. H. Press and D. N. Spergel, Capture by the sun of a galactic population of weakly interacting massive particles, Astrophys.J. 296 (1985) 679-684.

[7] L. Roszkowski, E. M. Sessolo and S. Trojanowski, WIMP dark matter candidates and searches current status and future prospects, Rept. Prog. Phys. 81 (2018) no.6, 066201, [arXiv:1707.06277 [hep-ph]].

[8] G. Jungman, M. Kamionkowsi and K. Griest, Supersymmetric Dark Matter, Phys. Rept. 267 (1996) 195, [arxiv:9506380 [hep-ph]].

[9] M. Masip, High energy neutrinos from the Sun, Astropart. Phys. 97 (2018) 63-68, [arXiv:1706.01290 [hep-ph]].

[10] M. Blennow, J. Edsjö and T. Ohlsson, Neutrinos from WIMP Annihilations Obtained Using a Full Three-Flavor Monte Carlo Approach, JCAP 0801, 021 (2008), [arXiv: 0709.3898].

[11] T. Chiarusi and M. Spurio, High-energy astrophysics with neutrino telescopes, Eur. Phys. J., $\mathbf{C 6 5}$ (2010) 649-701, [arXiv:0906.2634 [astro-ph.HE]].

[12] S. Adrián-Martínez, et al., Letter of Intent for KM3NeT 2.0, J.Phys. G43 (2016) no.8, 084001 [arXiv:1601.07459]. 
[13] M. Ageron, et al., ANTARES: The first undersea neutrino telescope, Nucl. Instum. Methods Phys. Res. A 656 (2011) 11. [arXiv:1104.1607].

[14] S. Adrián-Martínez, et al., The positioning system of the ANTARES Neutrino Telescope, J. Instrum. 7 (2012) T08002 [arXiv:1202.3894].

[15] R. Barlow, Extended Maximum Likelihood, Nucl. Instum. Methods Phys. Res. A 297 (1990) 496-506.

[16] S. Adrián-Martínez et al., Limits on dark matter annihilation in the sun using the ANTARES neutrino telescope, Phys. Lett. B 759 (2016) 69-74, [arXiv:1603.02228 [astro-ph.HE]].

[17] J. Edsjö, J. Elevant and C. Niblaeus, WimpSim Neutrino Monte Carlo, http://wimpsim.astroparticle.se/.

[18] M. Honda et al., Atmospheric Neutrino Flux Calculation Using the NRLMSISE-O0 Atmospheric Model, Phys. Rev., D 92 (2):023004, (2015) [arXiv:1502.03916 [astro-ph.HE]].

[19] J. Neyman, Outline of a Theory of Statistical Estimation Based on the Classical Theory of Probability, Phil. Trans. Royal Soc. London Series A.236, London 1937.

[20] G. Wikström and J. Edsjö, Limits on the WIMP-nucleon Scattering Cross-Section from Neutrino Telescopes, JCAP 0904 (2009) 009 [arXiv:0903.2986v2].

[21] J. Edsjö et al., http: / / www. darksusy. org.

[22] M. G. Aartsen et al., Search for annihilating dark matter in the Sun with 3 years of IceCube data, Eur.Phys.J. C 77 (2017) no.3, 146 [arXiv:1612.05949 [astro-ph.HE]].

[23] K. Frankiewicz, Searching for Dark Matter Annihilation into Neutrinos with Super-Kamiokande, Proceedings, Meeting of the APS Division of Particles and Fields (DPF 2015): Ann Arbor, Michigan, USA, 4-8 Aug 2015 [arXiv:1510.07999 [hep-ex]].

[24] C. Amole et al., Dark Matter Search Results from the PICO-60 $C_{3} F_{8}$ Bubble Chamber, Phys. Rev. Lett. 118 (2017) no.25, 251301 [arXiv:1702.07666 [astro-ph.CO]]. 